Pacific Journal of Mathematics

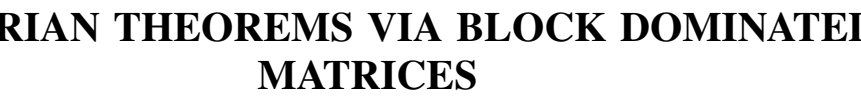




\section{TAUBERIAN THEOREMS VIA BLOCK DOMINATED MATRICES}

\section{J. A. FRIDY}

The principle result is a general Tauberian theorem that can be applied to any regular real matrix summability method. The Tauberian condition is determined by the lengths of the blocks of consecutive terms that dominate the rows of the matrix. This theorem and its variants are then used to give a unified method of proving some of the classical Tauberian theorems for the methods of Abel, Borel, and Euler-Knopp. The general technique is also used to prove new Tauberian theorems for Nörlund and Taylor matrices.

1. Introduction. Let $A$ be the matrix summability method that maps the sequence $x$ into the sequence $A x$, whose $n$th term is given by $(A x)_{n}=\sum_{k=0}^{\infty} a_{n k} x_{k}$. Let $\Delta x$ denote the sequence of differences: $(\Delta x)_{n}=x_{n}-x_{n+1}$. In this paper we shall study order-type Tauberian theorems in which the convergence of $A x$ together with a hypothesis such as $(\Delta x)_{n}=o\left(d_{n}\right)$ implies the convergence of $x$.

From each row of $A$ that is in $l^{1}$, we can choose a block of consecutive terms whose total dominates the row sum. In order to state this precisely, let us introduce some notation and terminology. For each $n$, let $B_{n}=\{k: \mu(n)<k \leqq \nu(n)\}$, where $\mu$ and $\nu$ are nonnegative integer sequences satisfying $\mu(n+1)<\nu(n)$. Note that every integer greater than $\mu(0)$ belongs to at least one block $B_{n}$. Let $L_{n}$ denote the length of $B_{n}$, i.e., $L_{n}=\nu(n)-\mu(n)$. We shall say that the matrix $A$ is $\left\{B_{n}\right\}$-dominated if

$$
\lim \inf _{n}\left\{\left|\sum_{k \in B_{n}} a_{n k}\right|-\sum_{k \notin B_{n}}\left|a_{n k}\right|\right\}>0 \text {. }
$$

The main result of this paper asserts that if $A$ is $\left\{B_{n}\right\}$-dominated, then $\max _{k \in B_{n}}\left|(\Delta x)_{k}\right|=o\left(L_{n}^{-1}\right)$ is a Tauberian condition for $A$ over the bounded sequences; if $A$ is rowfinite, then a similar assertion holds without the assumption that $x$ is bounded. The strength of this result lies not in its precision, but rather in its generality. It is easy to see that if $\lim \inf _{n}\left|\sum_{k=0}^{\infty} a_{n k}\right|>0$ and each row is in $l^{1}$, then $A$ admits a block sequence that satisfies (1). In case $A$ is regular, the sequence $\left\{B_{n}\right\}$ can be chosen satisfying (1) and $\lim _{n} \mu(n)=\infty$. This helps us to define $\left\{B_{n}\right\}$ so that $L_{n}$ does not increase too rapidly, thus obtaining a larger order estimate in the Tauberian condition. In $\S 3$ the dominant blocks concept is used as a unified approach to proving some classical Tauberian theorems for the methods of Abel, Borel, and Euler-Knopp. In the final section, new Tauberian theorems are proved for Nörlund 
and Taylor matrices.

2. General Tauberian theorems. The method of proof to be used here is suggested by an argument employed by Agnew [1] and Rado [8]. These authors considered matrices $A$ in which each row was dominated by a single term, and their conclusions were that $A$ is Mercerian, i.e., $A$ is equivalent to ordinary convergence. In case $A$ is $\left\{B_{n}\right\}$-dominated, we can use the slow oscillation of $x$ to make the terms of these dominant blocks coincide with $x_{k}$ 's whose values are all nearly equal to some limit point $\alpha$. Then $\sum_{k=0}^{\infty} a_{n k} x_{k}$ will approximate a multiple of $\alpha$, and in this way the convergence of $A x$ prevents $x$ from oscillating between two or more limit points.

Theorem 1. Suppose that $A$ is a regular real matrix that is $\left\{B_{n}\right\}$-dominated; if $x$ is a bounded sequence such that $A x$ is convergent and

$$
\max _{k \in B_{n}}\left|(\Delta x)_{k}\right|=o\left(L_{n}^{-1}\right)
$$

then $x$ is convergent.

Proof. We may assume that $x$ is a real number sequence. We suppose that $x$ is nonconvergent, and we shall show that no real number $r$ can be the limit of $A x$. Let $R$ denote the positive number $\lim \sup _{k}\left|x_{k}-r\right|$. Using the well-known Silverman-Töplitz conditions, we can write

$$
(A x)_{n}-r=o(1)+\sum_{k=0}^{\infty} a_{n k}\left(x_{k}-r\right) .
$$

Suppose $0<\varepsilon<R$, and choose $K$ such that $l \geqq K$ implies $\left|x_{k}-r\right|<$ $R+\varepsilon$. Then

$$
\begin{aligned}
\left|(A x)_{n}-r\right| & \geqq 0(1)+\left|\sum_{k \in B_{n}} a_{n k}\left(x_{k}-r\right)\right|-\sum_{k \notin B_{n}}\left|a_{n k}\right| \cdot\left|x_{k}-r\right| \\
& \geqq 0(1)+\left|\sum_{k \in B_{n}} a_{n k}\left(x_{k}-r\right)\right|-\sum_{\substack{k \notin B_{n} \\
k \geqq K}}\left|a_{n k}\right|\left|x_{k}-r\right| \\
& >0(1)+\left|\sum_{k \in B_{n}} a_{n k}\left(x_{k}-r\right)\right|-(R+\varepsilon) \sum_{k \in B_{n}}\left|a_{n k}\right| \\
& \geqq 0(1)+\left|\sum_{k \in B_{n}} a_{n i k}\left(x_{k}-r\right)\right|-R \sum_{k \in B_{n}}\left|a_{n k}\right|-\varepsilon\|A\|,
\end{aligned}
$$

where $\|A\|=\sup _{n} \sum_{k=0}^{\infty}\left|a_{n l}\right|$.

Next we assert that for infinitely many $n$,

$$
k \in B_{n} \text { implies }\left|x_{k}-r\right|>R-\varepsilon \text {. }
$$


This is established by first choosing $N$ so that $L_{n} \max _{i \in B_{n}}\left|(\Delta x)_{i}\right|<\varepsilon / 2$ whenever $n>N$; then select some $n$ greater than $N$ for which $B_{n}$ contains an integer $j$ satisfying $\left|x_{j}-r\right|>R-\varepsilon / 2$. For any $k$ in $B_{n}$, we have $\left|x_{k}-x_{j}\right| \leqq L_{n} \max _{i \in B_{n}}\left|(\Delta x)_{i}\right|<\varepsilon / 2$, so by the triangle inequality it follows that $\left|x_{k}-r\right|>R-\varepsilon$. Also, we note that all the numbers $\left\{x_{k}-r\right\}_{k \in B_{n}}$ have the same sign.

For any of the infinitely many $n$ as established in the preceding paragraph, we have

$$
\begin{aligned}
\left|(A x)_{n}-r\right| & \geqq o(1)+(R-\varepsilon)\left|\sum_{k \in B_{n}} a_{n k}\right|-R \sum_{k \notin B_{n}}\left|a_{n k}\right|-\varepsilon\|A\| \\
& \geqq o(1)-\varepsilon\left\{\|A\|+\left|\sum_{k \in B_{n}} a_{n k}\right|\right\}+R\left\{\left|\sum_{k \in B_{n}} a_{n k}\right|-\sum_{k: B_{n}}\left|a_{n k}\right|\right\} \\
& \geqq 0(1)-2 \varepsilon\|A\|+R\left\{\left|\sum_{k \in B_{n}} a_{n k}\right|-\sum_{k \notin B_{n}}\left|a_{n k}\right|\right\} .
\end{aligned}
$$

Since $\varepsilon$ is an arbitrarily small positive number, it follows from (1) that $\lim \inf _{n}\left|(A x)_{n}-r\right|>0$. Therefore, $A x$ cannot have limit $r$, whence $A x$ is nonconvergent.

The proof of Theorem 1 can be applied to sequences that satisfy a gap condition, say $(\Delta x)_{k}=0$, if $k \neq \kappa(m)$ where $m=0,1,2, \cdots$, instead of an order type Tauberian condition. In order to ensure that (3) holds we must assume that the block $B_{n}$ is contained in a single gap, i.e., $\kappa(m) \leqq \mu(n)<\nu(n) \leqq \kappa(m+1)$, for some $m$. The remainder of the proof can be used verbatim to yield the following general gap theorem. (Cf. [6, Theorem 1].)

COROLlary 1. Let $A$ be a regular real matrix that is $\left\{B_{n}\right\}-$ dominated and let $x$ be a bounded gap sequence such that $(\Delta x)_{n}=0$ if $k \neq \kappa(m), m=0,1, \cdots$. If $\left\{B_{n}\right\}$ and $\kappa$ satisfy $\kappa(m) \leqq \mu(n)<\nu(n) \leqq$ $\kappa(m+1)$ for infinitely many $n$, then $A x$ and $x$ either both converge or both diverge.

Although the assumption that $x$ is bounded places a considerable restriction on Theorem 1, we see by the following example that it is a necessary hypothesis. Let $A$ be the matrix given by

$$
a_{n k}=\left\{\begin{array}{cl}
3 / 2, & \text { if } k=n, \\
-1 / 2, & \text { if } k=n^{3}, \\
0, & \text { otherwise } .
\end{array}\right.
$$

It is clear that a dominant block sequence is $\left\{B_{n}\right\}=\{n\}$; thus $\mu(n)=$ $n-1, \nu(n)=n$, and $L_{n} \equiv 1$. Therefore, the Tauberian condition (2) reduces to $(\Delta x)_{n}=o(1)$. Consider the unbounded sequence $x: x_{n}=\log n$, if $n>0$. Then $\lim _{n}(\Delta x)_{n}=0$, and $(A x)_{n}=0$, if $n>0$, but obviously 
$x$ is not convergent.

In order to prove a result similar to Theorem 1 in which the hypothesis that $x$ is bounded is not necessary, we shall assume that $A$ is row finite and terms to the right of the dominant blocks are zero. The argument is an adaptation of the idea used by Agnew in $[1, \S 2]$.

Theorem 2. Suppose $A$ is a regular real matrix that is $\left\{B_{n}\right\}$ dominated and $a_{n k}=0$ whenever $k>\nu(n)$; if $x$ is a sequence such that $A x$ is convergent and $\max _{k \in B_{n}}\left|(\Delta x)_{k}\right|=o\left(L_{n}^{-1}\right)$, then $x$ is convergent.

Proof. Again, we assume that $x$ is real, and we suppose that $x$ is nonconvergent; by virtue of Theorem 1, we need to consider only the case where $x$ is unbounded. Let $H$ and $\varepsilon$ be arbitrary positive numbers, and choose $N$ such that $n>N$ implies that $L_{n} \max _{k k \in B_{n}}\left|(\Delta x)_{k}\right|<$ $\varepsilon$. Since $x$ is unbounded, we can select $n$ greater than $N$ for which $B_{n}$ contains an integer $p$ such that $\left|x_{p}\right|>\max _{k<p}\left\{\left|x_{k}\right|, H\right\}$. We also have $\max _{k \in B_{n}}\left|x_{k}-x_{p}\right|<\varepsilon$, which implies that for all $k$ in $B_{n},\left|x_{k}\right|>$ $\left|x_{p}\right|-\varepsilon$ and all $\left\{x_{k}\right\}_{k \in B_{n}}$ have the same sign. For such an $n$ we have

$$
\begin{aligned}
\left|(A x)_{n}\right| & \geqq\left|\sum_{k \in B_{n}} a_{n k} x_{k}\right|-\sum_{k \leqq \mu(n)}\left|a_{n k} x_{k}\right| \\
& >\left(\left|x_{p}\right|-\varepsilon\right)\left|\sum_{k \in B_{n}} a_{n k \mid}\right|-\sum_{k \leqq M(n)}\left|a_{n k} x_{p}\right| \\
& \geqq\left\{\left|\sum_{k \in B_{n}} a_{n k}\right|-\sum_{k \leqq M(n)}\left|a_{n k}\right|\right\} H-\varepsilon\|A\| .
\end{aligned}
$$

Since $H$ and $\varepsilon$ are arbitrary, it follows from (1) that $\lim \sup _{n}\left|(A x)_{n}\right|=$ $\infty$. Hence, $A x$ is nonconvergent.

As above, this theorem gives rise to a gap theorem, which we state next.

Corollary 2. Let $A$ be a regular real matrix that is $\left\{B_{n}\right\}$ dominated so that $a_{n i}=0$ whenever $k>\nu(n)$, and let $x$ be a gap sequence such that $(\Delta x)_{n}=0$ if $k \neq \kappa(m), m=0,1, \ldots$. If $\left\{B_{n}\right\}$ and $\kappa$ satisfy $\kappa(m) \leqq \mu(n)<\nu(n) \leqq \kappa(m+1)$ for infinitely many $n$, then $A x$ and $x$ either both converge or both diverge.

In order to apply Theorems 1 and 2 to particular classes of matrices, it will be helpful if we first make some observations and remarks establishing some relationships between the Tauberian condition (2) and the more customary order estimates $(\Delta x)_{k}=o\left(d_{k}\right)$ or $O\left(d_{l_{k}}\right)$. Suppose that $(\Delta x)_{k}=o\left(d_{k}\right)$, where $d$ is a nonincreasing sequence. Define $r_{n}=\max _{k>n}\left|(\Delta x)_{k}\right| d_{k} \mid$, so $\lim _{n} r_{n}=0$ and 


$$
L_{n} \max _{k \in B_{n}}\left|(\Delta x)_{k}\right| \leqq L_{n} d_{\mu(n)} \boldsymbol{r}_{\mu(n)} .
$$

For a particular matrix, if we can construct the blocks $\left\{B_{n}\right\}$ so that $L_{n} d_{\mu(n)}=O(1)$, then we can infer from (4) that (2) holds. These remarks and similar observations based on $(\Delta x)_{k}=O\left(d_{k}\right)$ can be summarized formally as follows:

Lemma 1. If $(\Delta x)_{k}=o\left(d_{k}\right)$, where $d$ is nonincreasing, and $A$ is $\left\{B_{n}\right\}$-dominated so that $\lim _{n} \mu(n)=\infty$ and $L_{n} d_{\mu(n)}=O(1)$, then (2) holds.

Lemma 2. If $(\Delta x)_{k}=O\left(d_{k}\right)$, where $d$ is nonincreasing, and $A$ is $\left\{B_{n}\right\}$-dominated so that $\lim _{n} \mu(n)=\infty$ and $L_{n} d_{n^{(n)}}=o(1)$, then (2) holds.

These lemmas provide some insight into the relationship between $o$ - and $O$-Tauberian conditions as well as the relative difficulty of working with the weaker $O$-condition. For example, if $d_{k}=k^{-1}$, as in Tauber's original theorem [9], then $L_{n} d_{\mu^{\prime}(n)}=\{\nu(n)-\mu(n)\} / \mu(n)$, which yields the implications

$$
(\Delta x)_{k}=o\left(k^{-1}\right) \quad \text { and } \quad \nu(n)=O(\mu(n)) \text { imply }
$$

and

$$
(\Delta x)_{k}=O\left(k^{-1}\right) \text { and } \nu(n) \sim \mu(n) \text { imply }(2) .
$$

For a given matrix, it is obviously easier to construct $\left\{B_{n}\right\}$ so that $\nu(n)=O(\mu(n))$ rather than $\nu(n) \sim \mu(n)$. Indeed, for the Cesàro matrix of order 1 , it is impossible for $\left\{B_{n}\right\}$ to satisfy both (1) and $\nu(n) \sim$ $\mu(n)$; but if we choose $\mu(n)=[n / 3]$ and $\nu(n)=n$, then $\left\{B_{n}\right\}$ satisfies (1) and $\nu(n)=O(\mu(n))$. The resulting $o$-Tauberian theorem will be proved in the next section for Cesàro matrices $C_{j}$ of all positive orders; our purpose in citing the $C_{1}$ case at this time is to demonstrate the fact that the general dominant block theory does not yield $O$-theorems. We must conclude, as did Lorentz [5, p. 228], that "these $O$-Tauberian theorems are connected with much more delicate properties of the matrix" than those made use of in Theorems 1 and 2 .

3. Applications to known Tauberian theorems. In this section we demonstrate how the block Tauberian results can be applied to some classical summability methods to derive well-known $o$-Tauberian theorems. (See e.g., [2] and [7].) First we consider the Cesàro matrix of order $j$, where $j$ is a positive integer, which is the lower triangular matrix given by

$$
C_{j}(n, k)=\left(\begin{array}{c}
n-k+j-1 \\
j-1
\end{array}\right)\left(\begin{array}{c}
n+j \\
j
\end{array}\right)^{-1} \quad(k \leqq n) .
$$


THEOREM 3. If the sequence $x$ is $C_{j}$-summable for some $j>0$ and $(\Delta x)_{k}=o\left(k^{-1}\right)$, then $x$ is convergent.

Proof. In order to apply Theorem 2, we must determine $\mu$ and $\nu$ so that (1) holds. First define $\nu(n)=n$. If $1 / 2<\beta<1$, we wish to define $\mu=\mu(n)$ so that $\sum_{k=\mu+1}^{n} C_{j}(n, k) \geqq \beta$. By manipulating binomial coefficients, we find that

$$
\begin{aligned}
\sum_{k=\mu+1}^{n} C_{j}(n, k) & =\left(1-\frac{\mu+1}{n+j}\right)\left(1-\frac{\mu+1}{n+j-1}\right) \cdots\left(1-\frac{\mu+1}{n+1}\right) \\
& \geqq\left(1-\frac{\mu+1}{n+1}\right)^{j} .
\end{aligned}
$$

Therefore, we want to have $\mu \leqq-1+(n+1)\left(1-\beta^{1 / j}\right)$, which is achieved by defining $\mu(n)=\left[n\left(1-\beta^{1 / j}\right)\right]$, where $[t]$ denotes the greatest integer not exceeding $t$. Since $\nu(n)=O(\mu(n))$, property (5) guarantees that (2) holds. Hence, by Theorem 2, $(\Delta x)_{k}=o\left(k^{-1}\right)$ is a Tauberian condition for $C_{j}$.

We remark that the preceding theorem applies to nonintegral $j$ as well, because $C_{j}$ includes $C_{r}$ whenever $0<r<j$.

Next we turn our attention to Abel summability, which assigns to a sequence $x$ the limit value $\lim _{t \rightarrow 1^{-}}\left\{(1-t) \sum_{k=0}^{\infty} x_{k} t^{k}\right\}$. Since the dominant blocks technique is designed for matrix methods, we replace the continuous parameter $t$ with a sequence $\left\{t_{n}\right\}$ such that $0<t_{n}<1$ and $\lim _{n} t_{n}=1$. Thus the Abel limit of $x$ is equal to $\lim _{n}(A x)_{n}$, where $A$ is the matrix given by $a_{n k}=\left(1-t_{n}\right) t_{n}^{k}$. It is clear that this Abel matrix method includes the continuous Abel method, so it also includes $C_{j}$ for every $j>-1$. The constraint is that $A$ is not row finite, and therefore, the Tauberian theorem that we can prove by using Theorem 1 will not apply to unbounded sequences.

THEOREM 4. If $x$ is a bounded sequence that is summable by a regular Abel matrix and $(\Delta x)_{k}=o\left(k^{-1}\right)$, then $x$ is convergent.

Proof. Suppose $\varepsilon>0$. Since $\sum_{k=\mu_{+1}}^{\nu} a_{n k}=t_{n}^{1+\mu}-t_{n}^{1+\nu}$, we first choose $\mu(n)$ as large as possible while still satisfying $t_{n}^{1+\mu(n)} \geqq 1 / 2+2 \varepsilon$, say $\mu(n)=\left[\log (1 / 2+2 \varepsilon) / \log t_{n}\right]$. Then choose $\nu(n)$ to be the least integer that is not less than $\log \varepsilon / \log t_{n}$. This yields

$$
\sum_{k \in B_{n}} a_{n k} \geqq \frac{1}{2}+\varepsilon,
$$

so (1) holds. Also

$$
\frac{\nu(n)}{\mu(n)} \sim \frac{\log \varepsilon}{\log \left(\frac{1}{2}+2 \varepsilon\right)}
$$


so $\nu(n)=O(\mu(n))$, and by property (5), we see that (2) holds. Hence, the assertion follows from Theorem 1.

As another application of Theorem 1, we shall give new proofs of the classical Tauberian theorems for the summability methods of Borel and Euler-Knopp. (See [2] and [3].) As above, we replace the continuous variable with a discrete one and work with the Borel matrix $B\left[7\right.$, p. 56], which is given by $b_{n k}=e^{-n} n^{k} / k$ !. The matrix $B$ includes the Borel exponential method, so the next result also applies to the latter method.

THEOREM 5. If $x$ is a bounded sequence that is summable by the Borel matrix and $(\Delta x)_{k}=o\left(k^{-1 / 2}\right)$, then $x$ is convergent.

Proof. It is sufficient to determine $\mu$ and $\nu$ satisfying (1) and $\{\nu(n)-\mu(n)\}\{\mu(n)\}^{-1 / 2}=O(1)$; from this, Lemma 1 will imply that (2) holds, and Theorem 1 then yields the conclusion. To simplify notation, let $u_{k}=n^{k} / k$ !. By a simple induction argument, one verifies that if $0 \leqq i \leqq \mu<n$, then $u_{i^{\prime-i}}<(\mu / n)^{i} u_{\mu}$. Thus

$$
\sum_{k=0}^{\mu} u_{k}<\sum_{i=0}^{\mu}\left(\frac{\mu}{n}\right)^{i} u_{\mu}<\frac{n u_{\mu}}{n-\mu},
$$

and therefore,

$$
\sum_{k=0}^{\mu} b_{n k}<\frac{e^{-n} n u_{\mu}}{n-\mu}<\frac{n}{(n-\mu) \sqrt{2 \pi n}} .
$$

It follows that $\sum_{k=0}^{\mu} b_{n k} \leqq \alpha<1 / 4$, if $\mu$ is defined by

$$
\mu(n)=\left[n-\left(\frac{n}{2 \pi \alpha^{2}}\right)^{1 / 2}\right] \text {. }
$$

The selection of $\nu$ is made from a similar calculation: if $i>0$ and $\nu>n$, then $u_{\nu+i}<(n / \nu)^{i} u_{\nu}$, which yields

$$
\sum_{k>\nu} u_{k}<\frac{n u_{\nu}}{\nu-n}
$$

and

$$
\sum_{k>\nu} b_{n k}<\frac{n}{(\nu-n) \sqrt{2 \pi n}} .
$$

As before, we want the sum to be no greater than $\alpha$, so we define $\nu(n)$ to be the least integer that is not exceeded by

$$
n+\left(\frac{n}{2 \pi \alpha^{2}}\right)^{1 / 2} \text {. }
$$


It is now easy to see that $\{\nu(n)-\mu(n)\}\{\mu(n)\}^{-1 / 2}=O(1)$, and $\sum_{k \in B_{n}} b_{n k} \geqq$ $1-2 \alpha>1 / 2$, for every $n$, so the proof is complete.

Since the matrix $B$ includes the Borel exponential method, which in turn includes the Euler-Knopp means [2, p. 183], the next result follows immediately from Theorem 5 .

THEOREM 6. If $x$ is a bounded sequence that is summable by a regular Euler-Knopp matrix and $(\Delta x)_{k}=o\left(k^{-1 / 2}\right)$, then $x$ is convergent.

4. Tauberian theorems for Nörlund and Taylor means. In the final section we shall apply the dominant block technique to prove some new Tauberian theorems for several well-known matrix methods. We first obtain a simple theorem for the Nörlund means $N_{p}$, which are given by

$$
N_{p}(n, k)=\left\{\begin{array}{cl}
p_{n-k} / P_{n}, & \text { if } k \leqq n, \\
0, & \text { if } k>n,
\end{array}\right.
$$

where $p$ is a nonnegative sequence such that $p_{0}>0$, and $P_{n}=\sum_{i=0}^{n} p_{i}$.

THEOREM 7. If the sequence $x$ is summable by $N_{p}$, where $p$ is in $l^{1}$, and $(\Delta x)_{k}=o(1)$, then $x$ is convergent.

Proof. For such $p, N_{p}$ is regular since $\lim _{n} p_{n} / P_{n}=0$. Let $M$ be the least positive integer such that $P_{M} \geqq(2 / 3) \sum_{i=0}^{\infty} p_{i}$. If $\nu(n)=n$ and $\mu(n)=\max \{0, n-M-1\}$, then $N_{p}$ is $\left\{B_{n}\right\}$-dominated and $L_{n} \leqq$ $M+1$ for every $n$. Thus $(\Delta x)_{k}=o(1)$ implies (2); so by Theorem 2, $(\Delta x)_{k}=o(1)$ is a Tauberian condition for $N_{p}$.

We remark that $(\Delta x)_{k}=o(1)$ is a Tauberian condition for any matrix that can be block dominated with $L_{n}$ bounded. In particular, if all the nonzero entries of $A$ lie on a finite number of diagonals, then $(\Delta x)_{k}=o(\mathbf{1})$ is a Tauberian condition for $A$.

In [4] Iyengar stated the following theorem, which was first proved by Varshney [10].

THEOREM 8. Let $p$ be a positive sequence such that $\left\{p_{n+1} / p_{n}\right\}$ increases to 1 and $P_{n} \rightarrow \infty$. If $x$ is summable by $N_{p}$ and $(\Delta x)_{l}=O\left(M^{-1}\right)$, where $M=M(n)$ is a positive integer defined by $P_{M} \leqq \beta P_{n}<P_{n+1}$, for some $\beta$ in $(0,1)$, then $x$ is convergent.

The next result that we shall prove is similar to Iyengar's theorem. Although we must assume a stronger Tauberian condition, the theorem covers a larger class of Nörlund means.

THEOREM 9. Let $p$ be a nonincreasing sequence and suppose $1 / 2<$ 
$\beta<1$. If $x$ is summable by $N_{p}$ and $(\Delta x)_{n}=o\left(M^{-1}\right)$, where $M=M(n)$ is the least integer such that $P_{M} \geqq \beta P_{n}$, then $x$ is convergent.

Proof. Define $\nu(n)=n$ and $\mu(n)=n-M-1$. Then

$$
\sum_{k \in B_{n}} N_{p}(n, k)=P_{n}^{-1} \sum_{i=0}^{M} p_{i} \geqq \beta,
$$

and $L_{n}=M+1$. It is not difficult to verify that $M$ is nonincreasing and $\lim _{n} \mu(n)=\infty$. Therefore, we shall be able to apply Lemma 1 using $d_{k}=M(k)^{-1}$, provided that $L_{n} M(\mu)^{-1}=O(1)$. To prove that this boundedness holds, we first note that because $p$ is nonincreasing, $M(k) / k$ is also nonincreasing. Therefore,

$$
\frac{L_{n}}{M(\mu)} \sim \frac{M(n) / n}{M(\mu) / \mu} \cdot \frac{n}{\mu} \leqq \frac{n}{\mu}=\left\{1-\left(\frac{M(n)}{n}-\frac{1}{n}\right)\right\}^{-1},
$$

for sufficiently large $n$. The fact that $M(n) / n$ is nonincreasing implies that $\lim \sup _{n}\{M(n) / n\}<1$, which establishes the boundedness of $L_{n} / M(\mu)$. Therefore, Lemma 1 guarantees that (2) holds, so Theorem 2 may be applied to complete the proof.

Finally we shall apply Theorem 1 to the Taylor means to get a Tauberian theorem of the usual order type. Recall that the Taylor matrix [7, p. 60] of order $r$ is given by

$$
t_{n k}=\left\{\begin{array}{cl}
\left(\begin{array}{l}
k \\
n
\end{array}\right) r^{k-n}(1-r)^{n+1}, & \text { if } k \geqq n, \\
0, & \text { if } k<n .
\end{array}\right.
$$

Note that if $|r|<1$, then the row sums of $T_{r}$ are identically equal to 1 because $(1-r)^{-n-1}=\sum_{k \geqq n}\left(\begin{array}{l}k \\ n\end{array}\right) r^{k-n}$.

THEOREM 10. If $0<r<1 / 2$ and $x$ is a bounded sequence such that $T_{r} x$ is convergent and $(\Delta x)_{k}=o\left(k^{-1}\right)$, then $x$ is convergent.

Proof. Since $T_{r}$ is an upper triangular matrix, we define $\mu(n)=n$ and wish to define $\nu=\nu(n)$ so that

$$
\lim \sup _{n} \sum_{k>\nu} t_{n k} \leqq \alpha<1 / 2 .
$$

In order to get a convenient estimate for $\sum_{k>\nu} t_{n k}$, we consider the MacLaurin series for the function

$$
f(r)=(1-r)^{-n-1}=\sum_{k \geqq n}\left(\begin{array}{l}
k \\
n
\end{array}\right) r^{k-n}=\sum_{j=0}^{\infty}\left(\begin{array}{c}
n+j \\
n
\end{array}\right) r^{j} .
$$

Thus $\sum_{k>\nu} t_{n k}$ is $(1-r)^{n+1}$ times the remainder term $R_{\nu-n}(r)$ of the 
last series. By straightforward calculation the Lagrange form of this remainder is found to be

$$
R_{\nu-n}(\vartheta)=\left(\begin{array}{l}
\nu \\
n
\end{array}\right)\left(1-\xi_{\nu-n}\right)^{-\nu-1} r^{\nu-n}
$$

where $0<\xi_{\nu-m}<r$. Since $\left(1-\xi_{\nu-n}\right)^{-\nu-1}<(1-r)^{-\nu-1}$, we have

$$
R_{\nu-n}(r)<\left(\begin{array}{c}
\nu \\
n
\end{array}\right)(1-r)^{-\nu-1} r^{\nu-n},
$$

which yields

$$
\begin{aligned}
\sum_{k>\nu} t_{n k} & =(1-r)^{n+1} R_{\nu-n}(r) \\
& <\left(\begin{array}{c}
\nu \\
n
\end{array}\right)(1-r)^{n-\nu} r^{\nu-n} \\
& =\left(\begin{array}{c}
\nu \\
n
\end{array}\right) \rho^{\nu-n},
\end{aligned}
$$

where $\rho=r(1-r)^{-1}$. Note that $\rho<1$ because $r<1 / 2$. Suppose that $\nu(n)=c n$, where $c$ is a positive integer to be specified below. Using Stirling's formula, we find that

$$
\left(\begin{array}{l}
\nu \\
n
\end{array}\right)=\frac{(c n) !}{(c n-n) ! n !} \sim \frac{(c n)^{c n}}{(c n-n)^{c n-n} n^{n}}\left\{\frac{c}{2 \pi n(c-1)}\right\}^{1 / 2},
$$

so

$$
\left(\begin{array}{l}
\nu \\
n
\end{array}\right) \rho^{\nu-n} \sim\left\{\frac{\rho c^{c /(c-1)}}{c-1}\right\}^{c n-n}\left\{\frac{c}{2 \pi n(c-1)}\right\}^{1 / 2} .
$$

Combining (8) and (9), we see that (7) will hold if

$$
\frac{\rho c^{c /(c-1)}}{c-1}<1
$$

which is equivalent to

$$
\rho<c^{1 /\left(1-c_{\rangle}\right.}-c^{c /(1-c)} .
$$

It is an elementary exercise to show that the right-hand member of (11) approaches 1 as $c \rightarrow \infty$. Since $\rho<1$, we can choose $c$ sufficiently large to guarantee that (10) holds. Hence, (7) is satisfied by the definition $\nu(n)=c n$. Also, $(\Delta x)_{k}=o\left(k^{-1}\right)$ by hypothesis, so we infer from (5) that (2) holds. Therefore, the hypotheses of Theorem 1 are satisfied, so we conclude that $x$ is convergent. 


\section{REFERENCES}

1. R. P. Agnew, Equivalence of methods for evaluation of sequences, Proc. Amer. Math. Soc., 3 (1952), 550-556.

2. G. H. Hardy, Divergent Series, Clarenden Press, Oxford, 1949.

3. G. H. Hardy and J. E. Littlewood, Theorems concerning summability of series by Borel's exponential method, Rendiconti Palermo, 41 (1916), 36-53.

4. K. S. K. Igengar, New convergence and summability tests for Fourier series, Proc. Indian Acad. Sci., 18A (943), 113-120.

5. G. G. Lorentz, Tauberian theorems and Tauberian conditions, Trans. Amer. Math. Soc., 63 (1948), 226-234.

6. G. G. Lorentz and K. L. Zeller, o- but not O-Tauberian theorems, Proc. Amer. Math. Soc., 45 (1974), 401-404.

7. R. E. Powell and S. M. Shah, Summability Theory and Its Applications, Van Nostrand Rheinhold, London, 1972.

8. R. Rado, Some elementary Tauberian theorems (I), Quart. J. Math., Oxford Ser., 9 (1938), 274-282.

9. A. Tauber, Ein Satz aus der Theorie der unendlichen Reihen, Monatsh. Math., 8 (1879), 273-277.

10. O. P. Varshney, On Iyengar's Tauberian theorem for Nörlund summability, Tôhoku Math. J., 16 (1964), 105-110.

Received June 12, 1978.

Kent State UNiversity

KENT, OH 44242 



\section{PACIFIC JOURNAL OF MATHEMATICS}

EDITORS

DONALD BABBITT (Managing Editor)

University of California

Los Angeles, California 90024

HUGo RossI

University of Utah

Salt Lake City, UT 84112

C. C. MOORE

J. DUGUNDJI

Department of Mathematics

University of Southern California

Los Angeles, California 90007

R. FinN AND J. MiLgraM

Stanford University

Stanford, California 94305

University of California

Berkeley, CA 94720

\section{ASSOCIATE EDITORS}

E. F. BrCKENBACH

B. H. NeUmanN

F. WOLF

K. YoshidA

\section{SUPPORTING INSTITUTIONS}

UNIVERSITY OF BRITISH COLUMBIA

UNIVERSITY OF SOUTHERN CALIFORNIA

CALIFORNIA INSTITUTE OF TECHNOLOGY

STANFORD UNIVERSITY

UNIVERSITY OF CALIFORNIA

UNIVERSITY OF HAWAII

MONTANA STATE UNIVERSITY

UNIVERSITY OF TOKYO

UNIVERSITY OF NEVADA, RENO

UNIVERSITY OF UTAH

NEW MEXICO STATE UNIVERSITY

WASHINGTON STATE UNIVERSITY

OREGON STATE UNIVERSITY

UNIVERSITY OF WASHINGTON

UNIVERSITY OF OREGON 


\section{Pacific Journal of Mathematics}

\section{Vol. 81, No. $1 \quad$ November, 1979}

Thomas E. Armstrong, Simplicial subdivision of infinite-dimensional compact cubes ..................................... 1

Herbert Stanley Bear, Jr., Approximate identities and pointwise convergence ................................

Richard David Bourgin, Partial orderings for integral representations on convex sets with the Radon-Nikodým property..................

Alan Day, Herbert S. Gaskill and Werner Poguntke, Distributive lattices

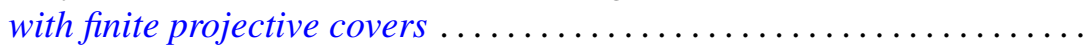

Heneri Amos Murima Dzinotyiweyi and Gerard L. G. Sleijpen, A note on measures on foundation semigroups with weakly compact orbits ......

Ronald James Evans, Resolution of sign ambiguities in Jacobi and Jacobsthal sums ...................................

John Albert Fridy, Tauberian theorems via block dominated matrices ......

Matthew Gould and Helen H. James, Automorphism groups retracting onto

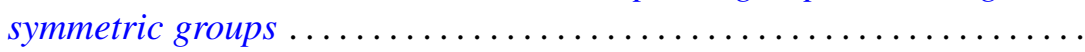

Kurt Kreith, Nonlinear differential equations with monotone solutions . . . . 101

Brian William McEnnis, Shifts on indefinite inner product spaces........ 113

Joseph B. Miles, On entire functions of infinite order with radially

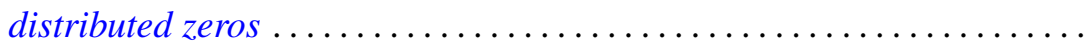

Janet E. Mills, The idempotents of a class of 0-simple inverse semigroups ...............................

Edward Jean Moulis, Jr., Generalizations of the Robertson functions ...

Richard A. Moynihan and Berthold Schweizer, Betweenness relations in probabilistic metric spaces.......................

Stanley Ocken, Perturbing embeddings in codimension two ....

Masilamani Sambandham, On the average number of real zeros of a class of random algebraic curves.

Jerry Searcy and B. Andreas Troesch, A cyclic inequality and a related eigenvalue problem.

Roger R. Smith and Joseph Dinneen Ward, $M$-ideals in $B\left(l_{p}\right)$...

Michel Talagrand, Deux généralisations d'un théorème de I. Namioka ..

Jürgen Voigt, $O n Y$-closed subspaces of $X$, for Banach spaces $X \subset Y$;

existence of alternating elements in subspaces of $C(J)$

Sidney Martin Webster, On mapping an $n$-ball into an $(n+1)$-ball in complex spaces

David J. Winter, Triangulable subalgebras of Lie p-algebras ... 